\title{
Tea polyphenols induce S phase arrest and apoptosis in gallbladder cancer cells
}

\author{
Jiaqi Wang ${ }^{1 *}$, Yixuan $\mathrm{Pan}^{1 *}$, Jiacheng $\mathrm{Hu}^{1 *}$, Qiang $\mathrm{Ma}^{2}$, Yi Xu ${ }^{2}$, Yijian Zhang ${ }^{2}$, Fei Zhang ${ }^{2}$ and \\ Yingbin Liu $^{2}$ \\ ${ }^{1}$ High School Affiliated Fudan University, Shanghai, China \\ ${ }^{2}$ Shanghai Research Center of Biliary Tract Disease, Shanghai, China
}

\begin{abstract}
Gallbladder cancer (GBC) is the most common malignancy in the biliary tract. Without effective treatment, its prognosis is notoriously poor. Tea polyphenols (TPs) have many pharmacological and health benefits, including antioxidant, antiinflammatory, anti-tumor, anti-thrombotic, antibacterial, and vasodilatory properties. However, the anti-cancer effect of TPs in human gallbladder cancer has not yet been determined. Cell viability and colony formation assay were used to investigate the cell growth. Cell cycle and apoptosis were evaluated by flow cytometry analysis. Western blot assay was used to detect the expression of proteins related to cell cycle and apoptosis. Human tumor xenografts were used to examine the effect of TPs on gallbladder cancer cells in vivo. TPs significantly inhibited cell growth of gallbladder cancer cell lines in a dose- and time-dependent manner. Cell cycle progression in GBC cells was blocked at the S phase by TPs. TPs also induced mitochondrial-related apoptosis in GBC cells by upregulating Bax, cleaved caspase-3, and cleaved PARP expressions and downregulating Bcl-2, cyclin A, and Cdk2 expressions. The effects of TPs on GBC were further proven in vivo in a mouse xenograft model. Our study is the first to report that TPs inhibit GBC cell growth and these compounds may have potential as novel therapeutic agents for treating gallbladder cancer.
\end{abstract}

Key words: Gallbladder cancer; Tea polyphenols; Apoptosis

\section{Introduction}

Gallbladder cancer (GBC) is the most common malignancy in the biliary tract and the sixth most common among gastrointestinal cancers (1-3), comprising $80-95 \%$ of biliary tract cancers worldwide. It is also the most aggressive biliary tract cancer, characterized by frequent chemo-resistance and recurrences after surgery. Currently, surgery is the most effective method for patients with GBC (4). However, many patients are diagnosed with advanced GBC with cancer cells that have been spread to lymph nodes or distal organs. For advanced stage GBC, surgical intervention is not possible (5), with unfavorable outcomes after chemotherapy or radiotherapy (6). Only $20 \%$ of patients respond to 5 -fluorouracil, and $36 \%$ respond to gemcitabine (7). The prognosis for patients of advanced GBC is poor, with 5-year survival rates from 20 to $40 \%$ (8). Therefore, it is important and necessary to find novel and effective therapy for GBC patients.

Tea polyphenols (TPs) are among the main components in green tea, and include epigallocatechin, epigallocatechin-3-gallate, epicatechin, and epicatechin-3-gallate. TPs have many pharmacological and health benefits including antioxidant, anti-inflammatory, anti-tumor, antithrombotic, antibacterial, and vasodilatory properties (9). TPs can induce apoptosis in various cancer cells, such as MCF-7 cells (10), bladder cancer SW780 cells (11), hepatoma HepG2 cells (12), and lung cancer H460 cells (13). TPs may mediate tumor cell apoptosis by regulating apoptosis-related proteins and signaling pathways; TPs

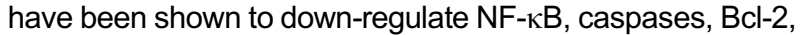
and stabilize p53 (14). In addition, TPs regulate cancer cell growth, survival, angiogenesis, and metastasis by MAPKs/ AP-1 and PI3K/Akt signaling pathway $(15,16)$.

Currently, however, there is a lack of data showing the effects of TPs in human gallbladder cancer. Here, we aimed to evaluate whether TP treatment inhibited the growth of various GBC cell lines.

\section{Material and Methods}

\section{Drugs and reagents}

TPs were obtained from the National Institute for the Control of Pharmaceutical and Biological Products (China).

Correspondence: Yingbin Liu: <liuyingbin@xinhuamed.com.cn> | Fei Zhang: <zhangfei36363636@163.com>

${ }^{*}$ These authors contributed equally to this study.

Received July 27, 2017| Accepted December 7, 2017 
The purity was greater than $95 \%$ as determined by highpressure liquid chromatography. TPs stock solution (40 mM) was prepared by dissolving TPs in dimethyl sulfoxide (DMSO). The stock solution was stored at $-20^{\circ} \mathrm{C}$. Dilutions of different concentrations were made using fresh complete medium before each treatment. DMSO was used as control. Antibodies against Bax, Bcl-2, Bad, cleavedPARP, cleaved-caspase 3, and GAPDH were purchased from Cell Signaling Technology (USA). CyclinA and CDK2 antibodies were purchased from Santa Cruz Biotechnology (USA). The antibody against Ki67 was purchased from Abcam (UK).

\section{Cell culture}

The SGC-996, NOZ, OCUG-1, and GBC-SD human gallbladder cancer cell lines were purchased from the Cell Bank of Type Culture Collection of the Chinese Academy of Sciences (China). Normal gallbladder epithelial cells (HGEpCs) were developed from normal gallbladder tissue (17). NOZ cells line was maintained in William's medium (Gibco, USA) supplemented with $10 \%$ fetal bovine serum (FBS; Gibco), $100 \mathrm{U} / \mathrm{mL}$ penicillin-streptomycin (Hyclone, USA) and glutamine at $4 \mathrm{mM}$. Dulbecco's Modified Eagle's Medium (DMEM; Gibco) containing 10\% FBS was used to maintain SGC-996, GBC-SD, OCUG-1, and HGEpC cell lines. A $37^{\circ} \mathrm{C}$ humidified incubator with $5 \% \mathrm{CO}_{2}$ was used to culture all the cell lines.

\section{Cell viability assay}

Cell viability was evaluated by the tetrazolium dye 3-(4,5-dimethylthiazol-2-yl)-2,5-diphenyltetrazolium bromide (MTT, Sigma-Aldrich, USA). GBC cells were seeded $(6 \times$ $10^{3}$ cells/well) into 96 -well plates and incubated at $37^{\circ} \mathrm{C}$ overnight. Cells were then treated with TPs solutions of different concentrations $(0,70,140,210,280$, or $360 \mu \mathrm{M})$ for 24,48 , or $72 \mathrm{~h}$. After each treatment time, $10 \mu \mathrm{L}$ of MTT reagent were added to each well $(0.5 \mathrm{mg} / \mathrm{mL})$ followed by 4-h incubation at $37^{\circ} \mathrm{C}$. Then the cell culture medium was discarded and supplemented with $200 \mu \mathrm{L}$ of DMSO. The absorbance at $490 \mathrm{~nm}$ was measured immediately using a 96-well plate ELISA reader (Bio-Rad Laboratories, USA).

\section{Colony formation assay}

GBC cells (500/well) were seeded and treated with TPs of different concentrations $(0,70$, or $140 \mu \mathrm{M})$ for $48 \mathrm{~h}$. After the treatment, the cells were maintained in culture medium for 10 days, allowing colony formation. The colonies were then fixed with $4 \%$ paraformaldehyde, followed by $0.1 \%$ crystal violet (Sigma-Aldrich) staining. The colonies were photographed using a microscope (Leica, Germany) and random fields were chosen for colony counting.

\section{Cell cycle analysis}

After being treated with TPs of different concentrations $(0,70$, or $140 \mu \mathrm{M})$ for $48 \mathrm{~h}, \mathrm{GBC}$ cells were trypsinized and fixed in $70 \%$ ethanol at $4{ }^{\circ} \mathrm{C}$ overnight. PI staining followed by flow cytometry analysis on a FACS Calibur system (Becton Dickinson, USA) was used to examine cell cycle. At least 50,000 stained cells for each sample were subjected to the analysis each time.

\section{Hoechst 33342 staining}

After being treated with TPs of different concentrations for $48 \mathrm{~h}$, the cells were washed in PBS and stained with $5 \mu \mathrm{g} / \mathrm{mL}$ Hoechst 33342 (Sigma-Aldrich) at $37^{\circ} \mathrm{C}$ in the dark for $10 \mathrm{~min}$. Nuclear DNA staining was observed using a fluorescence microscope. In each group, five microscopic fields were randomly selected and nuclear staining was counted.

\section{Annexin V/PI analysis}

GBC cells were treated with TPs of different concentrations $(0,70$, or $140 \mu \mathrm{M})$ for $48 \mathrm{~h}$. Apoptotic rate of cells was analyzed using an Annexin V/PI apoptosis kit (Invitrogen, USA). Staining and measuring procedures were performed according to the manufacturer's protocol.

\section{Mitochondrial membrane potential $(\Delta \Psi \mathrm{m})$ assay}

Fluorescent dye rhodamine 123 probe (Sigma-Aldrich) was used to analyze the mitochondrial membrane potential. After being treated with TPs of different concentrations for $48 \mathrm{~h}$ and washed twice with PBS, the cells were incubated with Rhodamine $123(5 \mu \mathrm{g} / \mathrm{mL})$ for $30 \mathrm{~min}$ at $37^{\circ} \mathrm{C}$. The final analysis was done by flow cytometry (Becton Dickinson).

\section{Western blot analysis}

After the treatment with TPs of different concentrations $(0,70$, or $140 \mu \mathrm{M})$ for $48 \mathrm{~h}, \mathrm{GBC}$ cells were harvested and total cellular proteins were extracted for western blot analysis. Protein concentrations were measured by BCA kit (Beyotime, China). Equal amounts of protein (50 ng) were loaded into $12 \%$ SDS-polyacrylamide gel for electrophoresis (SDS-PAGE). Nitrocellulose membranes were used for electro-transfer, which was performed at $300 \mathrm{~mA}$ for $2 \mathrm{~h}$. Five percent skim milk in Tris-buffered solution containing 1\% Tween-20 (TBST) was used to block the membrane. Membranes were then incubated with corresponding primary antibodies according to the protocols, followed by incubation with secondary antibodies at room temperature for $1 \mathrm{~h}$. A chemiluminescence (ECL) detection system was used for exposure.

\section{In vivo efficacy of TPs}

Six-week old BALB/c homozygous (nu/nu) nude mice (body weight of about $18 \mathrm{~g}$ ) were purchased from Shanghai SLAC Laboratory Animal Co., Ltd. (China). Tumor inoculation was performed in mice of 7 weeks of age. NOZ cells $\left(2 \times 10^{6}\right)$ suspended in $100 \mu \mathrm{L}$ PBS were injected into the right flank subcutis of the nude mice. The mice were randomly divided into 3 groups (4 mice/group): PBS (control), 20, or $40 \mathrm{mg} / \mathrm{kg}$ TPs were given to each group, 
respectively, via intraperitoneal (ip) injection every day for 5 weeks. After the 5 -week treatment, the mice were sacrificed and tumors were removed to determine the tumor sizes and weights. All animal procedures were conducted under guidance of the Care and Use of Laboratory Animals from National Institutes of Health. The animal protocol was approved by the Institutional Animal Care and Use Committee of Shanghai, Jiaotong University.

\section{Statistical analysis}

All results are reported as means $\pm S D$, calculated from at least three independent experiments. Differences between groups were analyzed using one- or two-way ANOVA with Dunnett's post hoc test. A P value of less than 0.05 was considered to be significant. SPSS software, v19.0 (SPSS Inc., USA) was used for all statistical analyses.
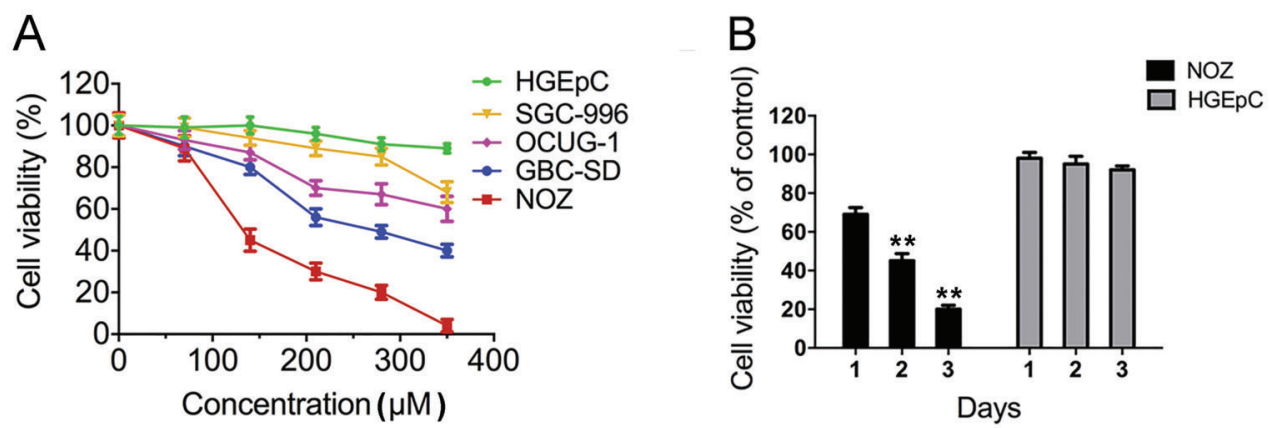

C
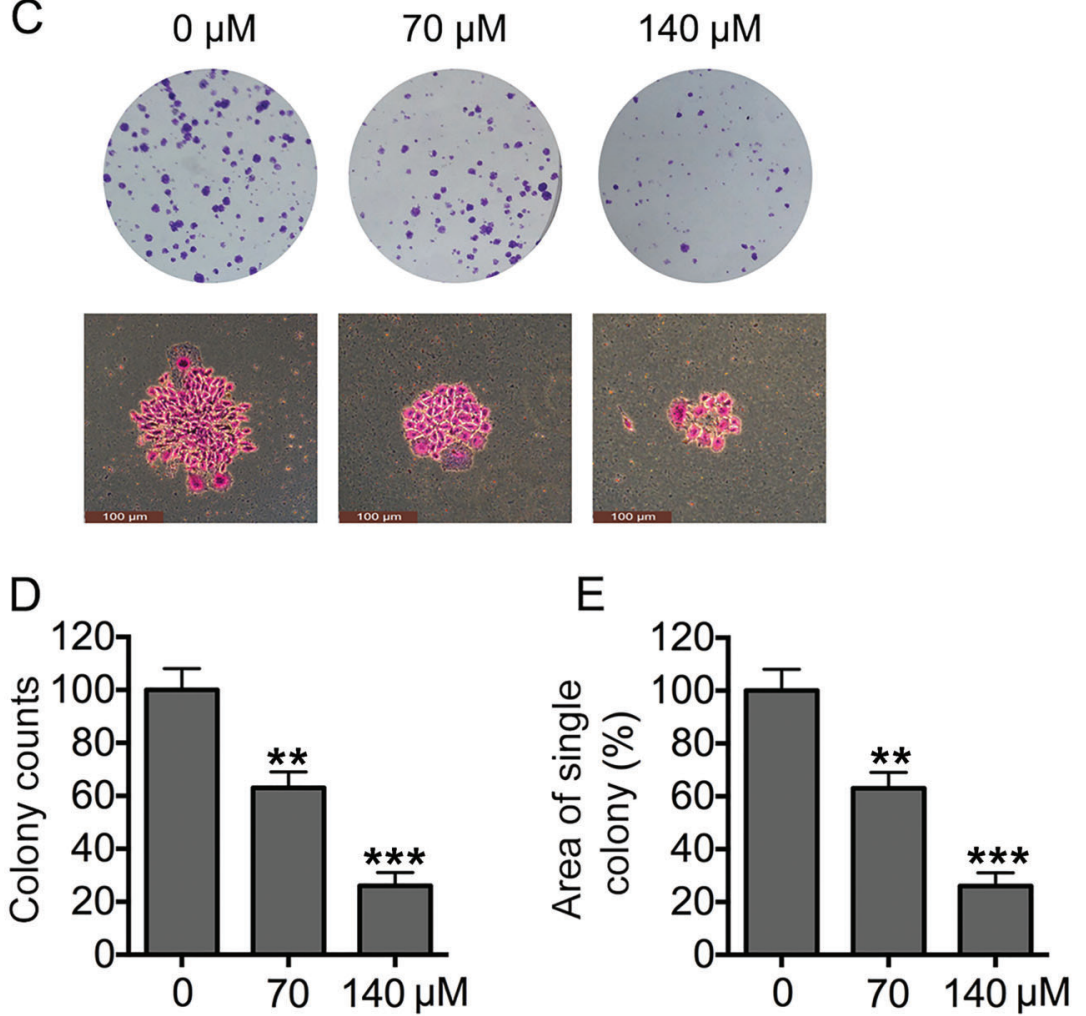

Figure 1. Tea polyphenols (TPs) inhibit gallbladder cancer (GBC) cell growth in a dose- and time-dependent manner. $A$, Four GBC cells and one normal gallbladder epithelial cell were assayed for cell viability after exposure to TP concentrations that ranged from 0 to $350 \mu \mathrm{M}$; untreated cells were used as controls. All the cells were cultured for $48 \mathrm{~h}$. $B$, At a fixed dose $(140 \mu \mathrm{M})$, TPs inhibited the viability of four GBC cells and one normal gallbladder epithelial cell in a time-dependent manner $(24,48$, or $72 \mathrm{~h})$. $C$, Colony formation assays were performed using NOZ cells treated with indicated doses of TPs. The colonies were stained with crystal violet. $D$, Colony formation was counted manually for each group of cells. $E$, The size of each colony was also calculated and averaged. ${ }^{* \star} P<0.01 ;{ }^{* \star} P<0.001$ compared to 0 (ANOVA). 


\section{Results}

TPs inhibited GBC cell growth in a dose- and time-dependent manner

The result showed that TPs had a dose- and timedependent killing effect on multiple GBC cell lines, but not on HGEpCs (Figure $1 A$ and $B$ ), indicating that TPs selectively kill GBC cells but not normal cells. The NOZ cell line was the most sensitive to TPs among four cell lines; TPs showed a significantly higher toxic effect on this cell line, with an $\mathrm{IC}_{50}$ value around $100 \mu \mathrm{M}$. Therefore, $\mathrm{NOZ}$ cell line was chosen for further functional analyses. $\mathrm{NOZ}$ cells were treated with TPs at concentrations from 0 to $140 \mu \mathrm{M}$ in vitro. Furthermore, we adopted the doses 0 , 70 , and $140 \mu \mathrm{M}$ of TPs due to the initial cytotoxicity results and subsequently used these concentrations to perform colony formation assays. It was visually observed that smaller colonies were formed as the TP dosage increased (Figure 1C). Quantitative analysis indicated that colony numbers decreased with increased TP dosage (Figure 1D). A smaller average growth area of a single colony was also observed with an increasing TP dosage (Figure 1E). These results suggest that TPs inhibit NOZ cell growth in a doseand time-dependent manner.

TPs induced $S$ phase arrest in GBC cells

TP treatment arrested the cell cycle in NOZ cells. More importantly, the percentage of cells in the G2/M phase decreased as the TP dosage increased. In contrast, there was an increased percentage of cells in the $S$ phase in TP-treated NOZ cells, compared to controls (Figure 2A). The results suggested that TPs can arrest the cell cycle at the $S$ phase in a dose-dependent manner. The result was further confirmed by western blotting, showing that S-G2/M related proteins cyclin A and CDK2 were down regulated in NOZ and GBC-SD cells after TP treatment (Figure 2B), consistent with the TP-induced S phase arrest. These results suggest that TPs induced $S$ phase arrest by regulating $S$ phase-related proteins cyclin $A$ and $C D K 2$ in GBC cells.

\section{TPs triggered mitochondrial-related apoptosis of GBC cells}

Chromatin condensation and fragmentation are two major indicators of cell apoptosis. In order to study the role of apoptosis in TPs mediated anti-cancer activities, we studied the nuclear morphology of TP-treated cells using Hoechst 33342 staining. The nuclei were stained homogeneously and weakly in blue in control cells, whereas some nuclear fragmentation and bright chromatin condensation were observed in TP-treated cells (Figure 3A). The amount of apoptotic nuclei indicated by bright chromatin condensation increased significantly as the TP concentration increased (Figure 3B). Apoptosis was confirmed by flow cytometry analysis of Annexin V-FITC/PI staining. As shown in Figure $3 \mathrm{~B}$, apoptosis in NOZ cells was increased at both
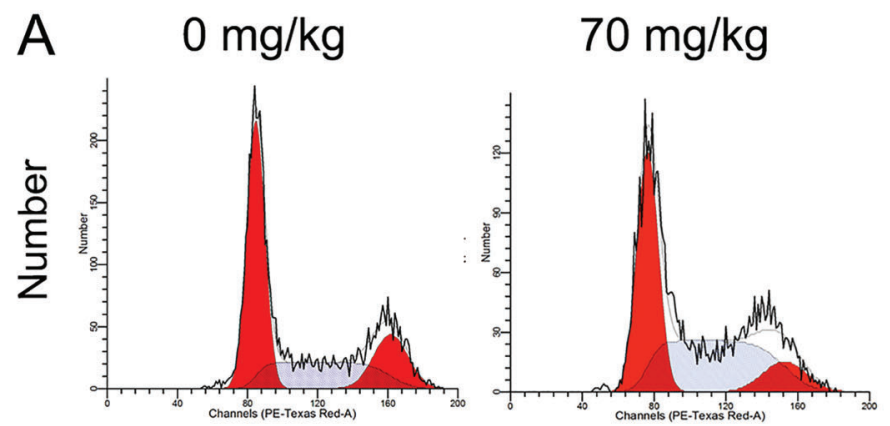

\section{$140 \mathrm{mg} / \mathrm{kg}$}
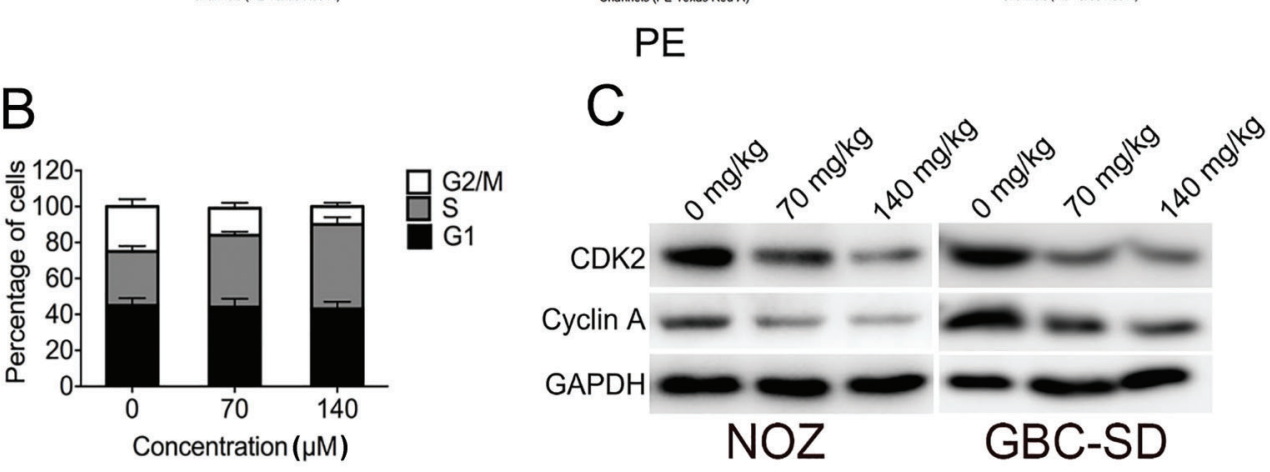

Figure 2. Tea polyphenols (TPs) induce $S$ phase arrest in gall bladder (GBC) cells. $A, B, N O Z$ cells without or with different TP treatment were subjected to cell cycle analysis by flow cytometry. $C$, Western blot analysis of cell cycle-related proteins in both cell lines. GAPDH was used as a loading control. Data are reported as means \pm SD of three independent experiments. 


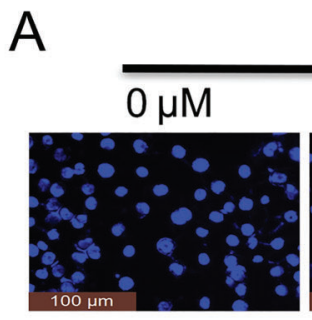

TP
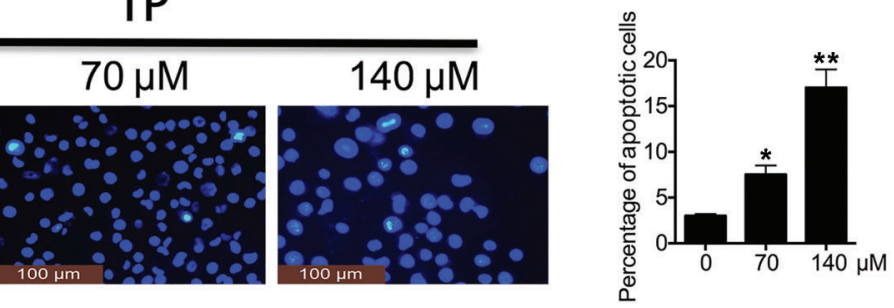

B

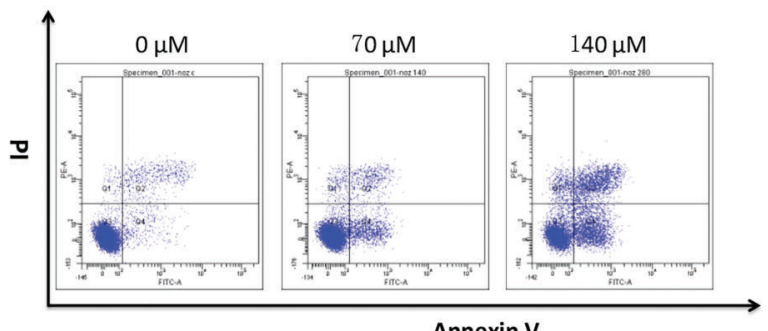

Annexin V
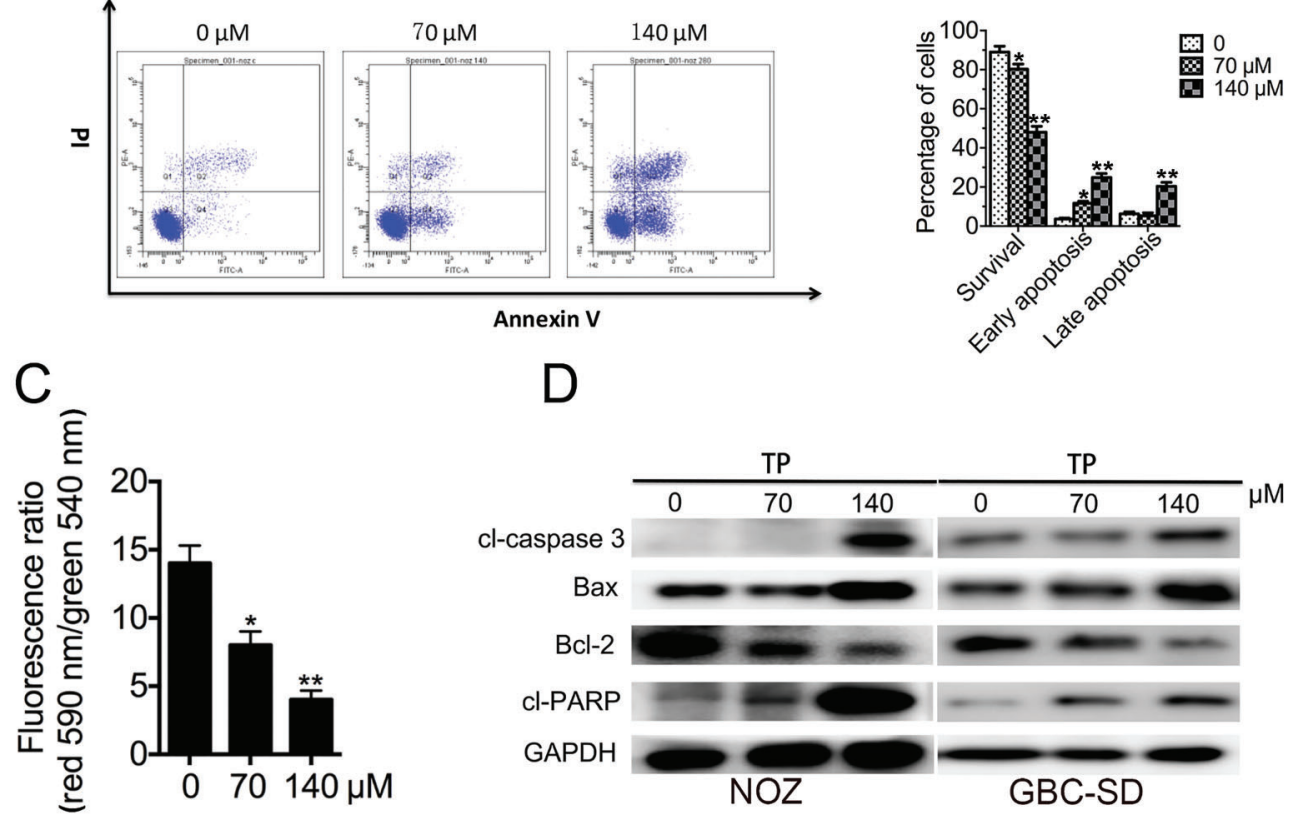

Figure 3. Tea polyphenols (TPs) trigger mitochondrial-related apoptosis of gallbladder cancer (GBC) cells. A, Changes in apoptotic nuclear morphology were observed by Hoechst 33342 staining and visualized by fluorescent microscopy. $B$, Cells were analyzed by flow cytometry with Annexin V-FITC/PI staining after TP treatment. Annexin V vs PI plots from the gated cells show the populations corresponding to viable (Annexin V-/PI-), necrotic (Annexin V-/PI+), early (Annexin V+/PI-), and late (Annexin V+/PI+) apoptotic

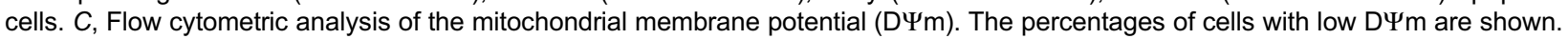
$D$, Western blot analysis of apoptosis-related proteins in both cell lines; GAPDH was used as a loading control. Data are reported as means $\pm S D$ of three independent experiments. ${ }^{*} \mathrm{P}<0.05$, ${ }^{* *} \mathrm{P}<0.01$ vs control (ANOVA).

early and late stages in a dose-dependent manner after TP treatment. These results strongly suggest that TP-induced inhibition of cell growth coincides with increased apoptosis in GBC cells. To further determine whether the apoptosis was mitochondria-dependent, we examined the mitochondrial membrane potential in TPs treated cells. Compared to controls, TP treatment caused an obvious, dose-dependent decrease in the mitochondrial membrane potential in NOZ cells (Figure 3C). Apoptosis is a caspase-dependent programmed cell death; a significant proteolytic cleavage of caspase-3 and PARP were observed in TPs treated NOZ cells. Furthermore, the level of pro-apoptotic mitochondrial protein Bax was increased, whereas the level of antiapoptotic protein $\mathrm{Bcl}-2$ was decreased in TPs treated NOZ and GBC-SD cells (Figure 3D). These results indicate that
TPs can induce apoptosis through mitochondrial signaling pathways in GBC cells.

\section{TPs inhibited tumor growth in a xenograft model of GBC}

A xenograft model of GBC was established using $\mathrm{NOZ}$ and GBC-SD cells. Mice were injected with TPs (20 or $40 \mathrm{mg} / \mathrm{kg}$ ) daily for 5 weeks; mice that were injected with PBS were used as controls. The tumor volumes were different three weeks after inoculation. After five weeks, the mice in the control group exhibited the largest tumor volumes, whereas mice treated with TPs exhibited significantly smaller tumor sizes than the control group (Figure 4A). After dissection, the tumor sizes were significantly larger in control mice than TP-treated mice (Figure 4B). 


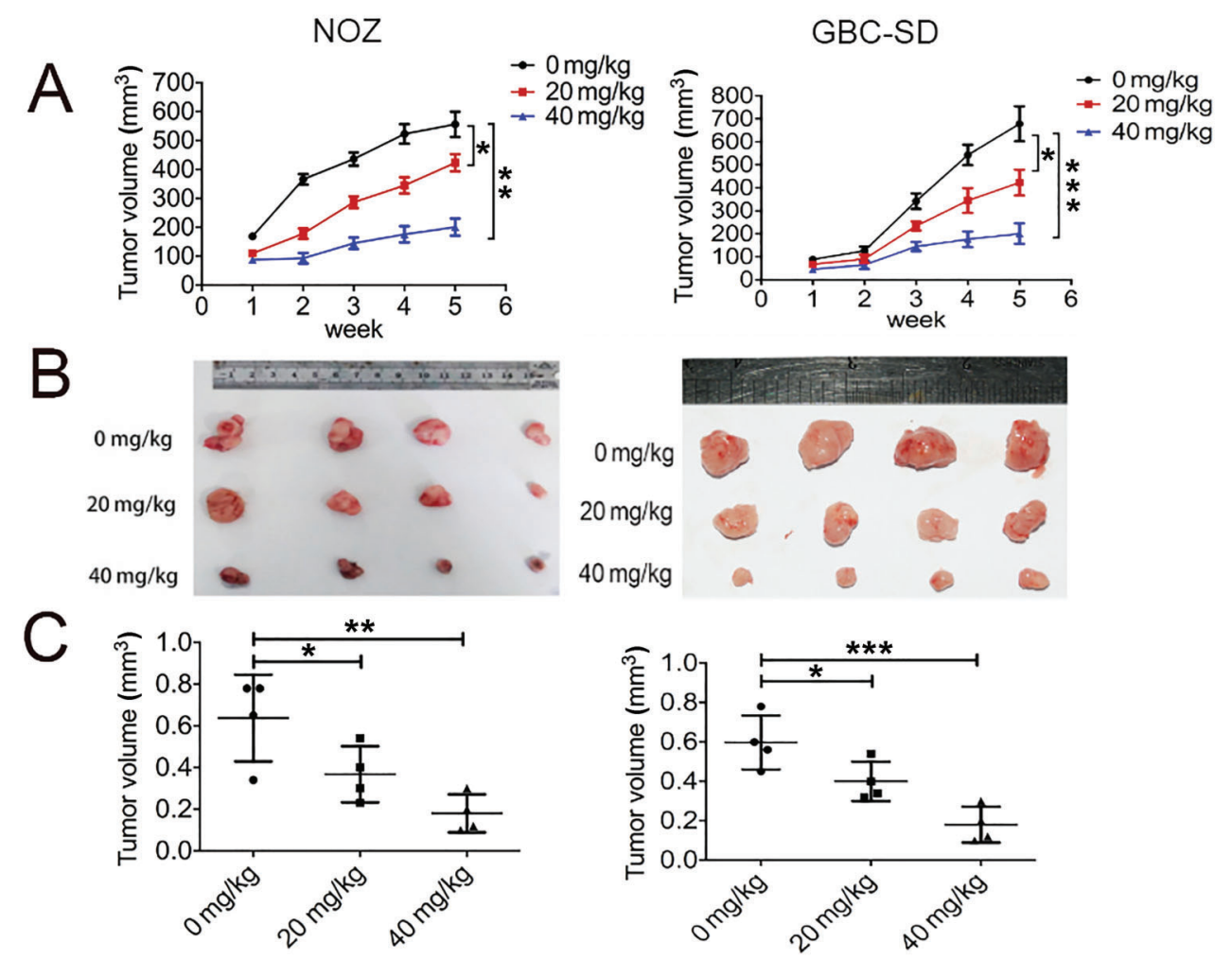

D

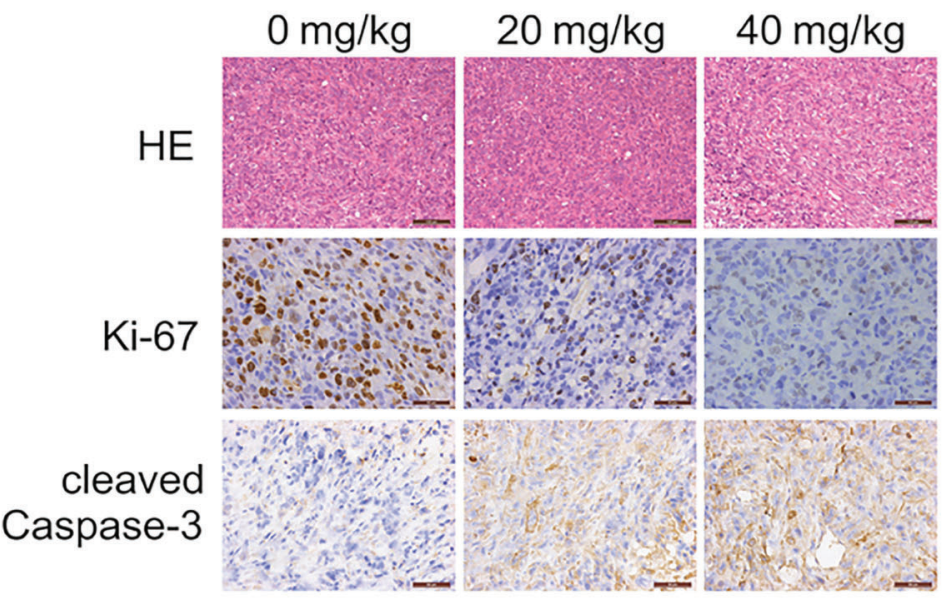

Figure 4. Tea polyphenols (TPs) effect on tumor growth in a xenograft model of gallbladder cancer (GBC). $A$, Volumes of dissected tumors during 6 weeks. $B$, Photographs of dissected tumors from each group after 7 weeks. $C$, Comparison of tumor volumes between groups. $D$, Expression of $\mathrm{Ki}-67$ and cleaved-Caspase-3 was detected in xenografts by IHC. Data are reported as means $\pm S D$. ${ }^{*} P<0.05$; ${ }^{* *} \mathrm{P}<0.01$ compared to control (ANOVA).

Tumor weights were significantly different among the three groups as well. The average weight of tumor in mice injected with 20 and $40 \mathrm{mg} / \mathrm{kg}$ of TPs was only 65.4 and $56.7 \%$ of that in control mice, respectively. Treatment with $40 \mathrm{mg} / \mathrm{kg}$ of TPs remarkably shrunk the tumors compared to those in control group (Figure 4C). Furthermore, IHC staining showed decreased levels of proliferating cell nuclear antigen (Ki67), indicating significantly fewer proliferative cells, and increased levels of cleaved caspase-3, indicating significantly more apoptotic cells in TP-treated xenograft tumors than in control tumors (Figure 4D). These results suggest that TPs can inhibit GBC growth in vivo. 


\section{Discussion}

GBC is the 6th-most common digestive tract malignancy worldwide (18). There is lack of effective therapy available for advanced GBC at present, and it is important to discover new therapies. Chinese herbal medicines have been shown to have effective antitumor activities with minimal toxicities. Therefore, they could be valuable candidates for developing novel chemotherapeutic agents.

TPs suppress tumors by inhibiting cell proliferation (19) and promoting tumor cell apoptosis (20). Targeting the molecules involved in apoptosis may provide new ideas for the search and development of anti-tumor drugs. TPs were involved in regulating a variety of signaling pathways in apoptosis in tumor cells. TPs inhibit the activity of protein kinase $\mathrm{C}$ and $\mathrm{CDKs}$, upregulating the expression of $\mathrm{lkBa}$, and initiating caspase cascades in mitochondria (21). In addition, TPs exert their chemoprevention effects by blocking proliferation and differentiation through modulating EGFR-MAPK signaling pathway and regulating c-jun, c-fos, and c-myc expression (22).

In this study, GBC cell growth was significantly suppressed by TPs both in vivo and in vitro. We further examined the effects of TPs on apoptosis and cell cycle arrest in GBC cells, since they are two major indicators of anticancer activities in cancer cells. The results suggested that TPS could arrest GBC cell cycle at $S$ phase and induce its mitochondria-related apoptosis. Moreover, the results showed that TPs had no toxic effects on normal gallbladder cells. Therefore, our results indicated that TPs is a safe molecule with a significant potential for GBC treatment clinically.

Apoptosis is a programmed cell death, which plays a critical role in development and health maintenance (9). Mitochondria-related apoptosis is the major apoptotic pathways in cancer cells (23). A study showed that TPS

\section{References}

1. Zhang F, Li M, Wu X, Hu Y, Cao Y, Wang X, et al. 20(S)ginsenoside $\mathrm{Rg} 3$ promotes senescence and apoptosis in gallbladder cancer cells via the p53 pathway. Drug Des Devel Ther 2015; 9: 3969-3987.

2. Li M, Zhang S, Wang Z, Zhang B, Wu X, Weng H, et al. Prognostic significance of nemo-like kinase (NLK) expression in patients with gallbladder cancer. Tumour Biol 2013; 34: 3995-4000, doi: 10.1007/s13277-013-0988-4.

3. Zhang F, Xiang S, Cao Y, Li M, Ma Q, Liang H, et al. EIF3D promotes gallbladder cancer development by stabilizing GRK2 kinase and activating PI3K-AKT signaling pathway. Cell Death Dis 2017; 8: e2868, doi: 10.1038/cddis.2017.263.

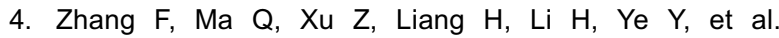
Dihydroartemisinin inhibits TCTP-dependent metastasis in gallbladder cancer. J Exp Clin Cancer Res 2017; 36: 68, doi: 10.1186/s13046-017-0531-3. mediated tumor cell apoptosis by regulating apoptosisrelated proteins and signaling pathways. TPs have been shown to down-regulate NF-KB, caspases, and $\mathrm{Bcl}-2$, and stabilize p53 (14). In addition, TPs regulate cancer cell growth, survival, angiogenesis and metastasis by MAPKs/ AP-1 and PI3K/Akt signaling pathway $(15,16)$. TP also promoted apoptosis in cancer cells in both the presence and absence of p53 function, through the survival signaling pathways that converge in the execution of apoptosis through involvement of the mitochondrial death cascade (19).

In our study, we found that treating NOZ cells with TPs for $48 \mathrm{~h}$ decreased the mitochondrial membrane potential, suggesting that the mitochondrial-related apoptosis played an important role in TPs induced apoptosis of gallbladder cancer cells. To further explore the mechanism of the TPinduced mitochondrial-related apoptosis, we detected the cell expression levels of Bcl2, Bax, cleaved caspase-3, and cleaved PARP. Our data showed that Bax expression was increased and $\mathrm{Bcl} 2$ expression was decreased after TP treatment. The change of the protein expression profile suggested a reduction in mitochondrial membrane potential and an increase in cytochrome $c$ releasing into the cytosol as a result. Our results also showed that TPs treatment can increase the expression of cleaved caspase-3 and cleaved PARP. These proteins belong to the Caspases family, which is involved in the downstream events of mitochondrialmediated apoptosis $(24,25)$. Our results suggested that TPs treatment induces mitochondrial-mediated apoptosis by activating the caspase-3 and PARP apoptotic cascade. TPs-induced apoptosis in GBC cells occurred through the mitochondrial pathway.

Taken together, the current study provides for the first time evidence that TPs can induce cell cycle arrest and promote apoptosis in GBC cells. The anticancer activities of TPs suggest that they are a promising candidate for the development of novel therapeutic reagents against GBC.
5. Cao $\mathrm{Y}$, Liang $\mathrm{H}$, Zhang $\mathrm{F}$, Luan $\mathrm{Z}$, Zhao $\mathrm{S}$, Wang $X A$, et al. Prohibitin overexpression predicts poor prognosis and promotes cell proliferation and invasion through ERK pathway activation in gallbladder cancer. $J$ Exp Clin Cancer Res 2016; 35: 68, doi: 10.1186/s13046-016-0346-7.

6. Cao Y, Liu X, Lu W, Chen Y, Wu X, Li M, et al. Fibronectin promotes cell proliferation and invasion through mTOR signaling pathway activation in gallbladder cancer. Cancer Lett 2015; 360: 141-150, doi: 10.1016/j.canlet.2015.01.041.

7. Dutta U. Gallbladder cancer: can newer insights improve the outcome? J Gastroenterol Hepatol 2012; 27: 642-653, doi: 10.1111/j.1440-1746.2011.07048.x.

8. Gold DG, Miller RC, Haddock MG, Gunderson LL, Quevedo F, Donohue $\mathrm{JH}$, et al. Adjuvant therapy for gallbladder carcinoma: the Mayo Clinic Experience. Int J Radiat Oncol Biol Phys 2009; 75: 150-155, doi: 10.1016/j.jijobp.2008.10.052. 
9. Gormaz JG, Valls N, Sotomayor C, Turner T, Rodrigo R. Potential role of polyphenols in the prevention of cardiovascular diseases: molecular bases. Curr Med Chem 2016; 23: 115-128, doi: 10.2174/0929867323666151127 201732.

10. Liu SM, Ou SY, Huang HH. Green tea polyphenols induce cell death in breast cancer MCF-7 cells through induction of cell cycle arrest and mitochondrial-mediated apoptosis. J Zhejiang Univ Sci B 2017; 18: 89-98, doi: 10.1631/jzus. B1600022.

11. Luo KW, Wei C, Lung WY, Wei XY, Cheng BH, Cai ZM, et al. EGCG inhibited bladder cancer SW780 cell proliferation and migration both in vitro and in vivo via down-regulation of NF-kappaB and MMP-9. J Nutr Biochem 2017; 41: 56-64, doi: 10.1016/j.jnutbio.2016.12.004.

12. Liang J, Li F, Fang Y, Yang W, An X, Zhao L, et al. Cytotoxicity and apoptotic effects of tea polyphenol-loaded chitosan nanoparticles on human hepatoma HepG2 cells. Mater Sci Eng C Mater Biol Appl 2014; 36: 7-13, doi: 10.1016/ j.msec.2013.11.039.

13. Hessien M, El-Gendy S, Donia T, Sikkena MA. Growth inhibition of human non-small lung cancer cells h460 by green tea and ginger polyphenols. Anticancer Agents Med Chem 2012; 12: 383-390, doi: 10.2174/18715201280022 8698.

14. Alshatwi AA, Periasamy VS, Athinarayanan J, Elango R. Synergistic anticancer activity of dietary tea polyphenols and bleomycin hydrochloride in human cervical cancer cell: Caspase-dependent and independent apoptotic pathways. Chem Biol Interact 2016; 247: 1-10, doi: 10.1016/j.cbi.2016. 01.012.

15. Singh BN, Shankar S, Srivastava RK. Green tea catechin, epigallocatechin-3-gallate (EGCG): mechanisms, perspectives and clinical applications. Biochem Pharmacol 2011; 82: 1807-1821, doi: 10.1016/j.bcp.2011.07.093.

16. Kanwar J, Taskeen M, Mohammad I, Huo C, Chan TH, Dou QP. Recent advances on tea polyphenols. Front Biosci 2012; 4: 111-131, doi: 10.2741/e363.
17. Ali MY, Anand SV, Tangella K, Ramkumar D, Saif TA. Isolation of Primary human colon tumor cells from surgical tissues and culturing them directly on soft elastic substrates for traction cytometry. J Vis Exp 2015: e52532.

18. Li M, Zhang Z, Li X, Ye J, Wu X, Tan Z, et al. Whole-exome and targeted gene sequencing of gallbladder carcinoma identifies recurrent mutations in the ErbB pathway. Nat Genet 2014; 46: 872-876, doi: 10.1038/ng.3030.

19. Gupta K, Thakur VS, Bhaskaran N, Nawab A, Babcook MA, Jackson MW, et al. Green tea polyphenols induce p53dependent and p53-independent apoptosis in prostate cancer cells through two distinct mechanisms. PLoS One 2012; 7: e52572, doi: 10.1371/journal.pone.0052572.

20. Halder B, Das Gupta S, Gomes A. Black tea polyphenols induce human leukemic cell cycle arrest by inhibiting Akt signaling: possible involvement of Hsp90, Wnt/beta-catenin signaling and FOXO1. FEBS $J$ 2012; 279: 2876-2891, doi: 10.1111/j.1742-4658.2012.08668.x.

21. Monobe M, Ema K, Tokuda Y, Maeda-Yamamoto M. Enhancement of phagocytic activity of macrophage-like cells by pyrogallol-type green tea polyphenols through caspase signaling pathways. Cytotechnology 2010; 62: 201-203, doi: 10.1007/s10616-010-9280-2.

22. Lin JK. Cancer chemoprevention by tea polyphenols through modulating signal transduction pathways. Arch Pharm Res 2002; 25: 561-571, doi: 10.1007/BF02976924.

23. Basanez G, Soane L, Hardwick JM. A new view of the lethal apoptotic pore. PLoS Biol 2012; 10: e1001399, doi: 10.1371/ journal.pbio.1001399.

24. Lin $\mathrm{Y}, \mathrm{Xu}$ J, Liao H, Li L, Pan L. Piperine induces apoptosis of lung cancer A549 cells via p53-dependent mitochondrial signaling pathway. Tumour Biol 2014; 35: 3305-3310, doi: 10.1007/s13277-013-1433-4.

25. Zhang F, Liu B, Wang Z, Yu XJ, Ni QX, Yang WT, et al. A novel regulatory mechanism of Pim-3 kinase stability and its involvement in pancreatic cancer progression. Mol Cancer Res 2013; 11: 1508-1520, doi: 10.1158/1541-7786.MCR-130389. 